\title{
SETHI : Digital radar system for signal generation and acquisition
}

\author{
Jérôme HENRION \\ DEMR \\ ONERA \\ F-13661 Salon cedex Air - France \\ jerome.henrion@onera.fr
Nicolas CASTET
$D E M R$ \\ ONERA \\ F-13661 Salon cedex Air - France \\ nicolas.castet@onera.fr
}

\author{
Olivier BOISOT \\ DEMR \\ ONERA \\ F-13661 Salon cedex Air - France \\ olivier.boisot@onera.fr \\ Jean-François NOUVEL \\ DEMR \\ ONERA \\ F-13661 Salon cedex Air - France \\ jean-francois.nouvel@onera.fr
}

\author{
Rémi Baqué \\ DEMR \\ ONERA \\ F-13661 Salon cedex Air - France \\ remi.baque@onera.fr \\ Olivier Ruault du Plessis \\ DEMR \\ ONERA \\ F-13661 Salon cedex Air - France \\ Olivier.Ruault_du_Plessis@onera.fr
}

\begin{abstract}
SETHI is an airborne SAR system developed by ONERA, and integrating various sensors. The microwave ones cover from VHF-UHF to $X$ or Ku Band, full polarimetric and very high resolution for high quality and advanced applications like interferometry and tomography applications. ONERA invest a lot in the entire SETHI platform, and particularly in the new digital system used since 2017, called Système d'Acquisition et de Mesures Expérimentales (SAMEVA). This new system gives us a lot of new perspectives. It is based on a modular PXI platform (PCI eXtension for Instrumentation) which gives us flexibility, modularity, quality and reliability. We also have some real-time streaming capabilities, GPS timestamping and the driving capacity of the entire analogic radar system. This paper presents mainly the digitizers and the new arbitrary waveform generation based on a VST (Vector Signal Transceiver)
\end{abstract}

Keywords-digital, synchronization, modularity, highbandwidth, digitizers, FPGA, streaming, GPS, VST, signal, SAR

\section{INTRODUCTION}

SETHI is a SAR system developed by ONERA. It combines two pods under the wings which are able to carry different kinds of radar heads, ranging from VHF/UHF to Ku band and/or optical sensors, and allows a wide range of acquisition geometries [1]. The pod-based concept allows an easy integration and testing of new systems under the single certification of the pods issued by the authorities.

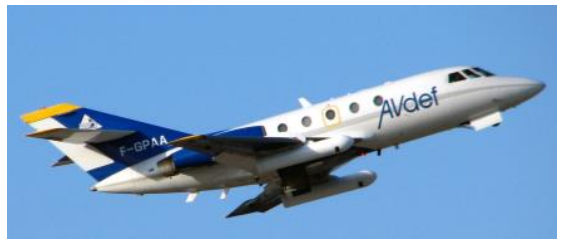

Fig. 1. Falcon 20 with pods after take off.

SETHI is integrated onboard a Falcon 20 (fig. 1) designed by Dassault Aviation, and operated by AVDEF (a French company based at Nîmes airport).

\section{SAMEVA}

Système d'Acquisition et de Mesures Expérimentales (SAMEVA) is our new digital acquisition and generation system based on the PXI platform. You can see below (Fig. 2) the full National Instruments (NI) chassis system with multiple cards and instruments inside.

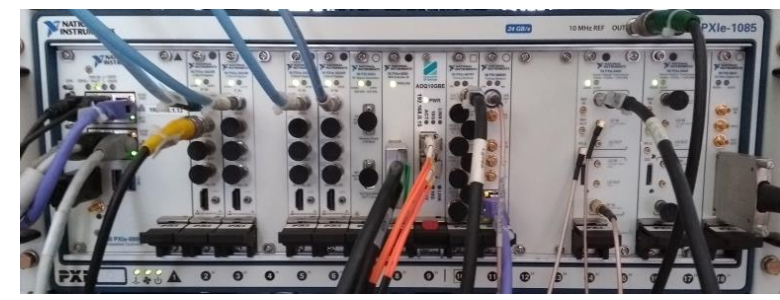

Fig. 2. SAMEVA Chassis PXIe-1085 from National Instruments

Fig. 2: Depicts the 18-slots chassis with integrated power supply containing, from left to right:

- the controller part, equivalent to a computer, with the Central Processing Unit (CPU), Solid State Drive (SSD) and embedded RAM

- Four IF-digitizers of $800 \mathrm{MHz}$ analog bandwidth

- Two Vector Signal Transceivers (VST)

The current SETHI configuration uses only the generation part of the two VSTs.

In a $4 \mathrm{U}$ form factor, we can have more than four synchronized digitizers and two waveform generators with a large instantaneous bandwidth, very good spectral purity and an excellent synchronization. Backplane clocks and trigger lines are really important for the whole system to be correctly synchronized. This PXI platform allows having a very good flexibility. Depending on the acquisitions configuration, we can add or remove instruments easily. 


\section{IF-DIGITIZERS}

Currently we are using four single channel IF-digitizers. The configuration is made by pair, each of which dealing with one frequency band, for example X-band for the first pair and Ku-band for the other. Each pair receives Horizontal and Vertical polarization simultaneously for the associated band. Fig. 3 illustrates the aforementioned digitizer card.

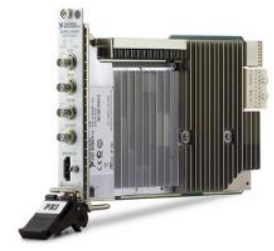

Fig. 3. Illustration of a NI PXIe-5624R IF-Digitizer

\section{A. Windowing FPGA personnality}

The NI PXIe-5624R IF-Digitizer is based on a programmable Kintex-7 FPGA allowing customizing the instrument. For SAR applications, the digitization of a few

samples within the complete PRI (Period Repetition Interval) is necessary and is easily pickable from the customization ability of the FPGA. Fig. 4 depicts our very flexible multirecord system allowing to choose multiple windows within a single PRI. Usually we are using three windows of acquisition; the first one is used for the reinjection (full system loopback), the second for the measure of noise, and the last one for the useful signal.

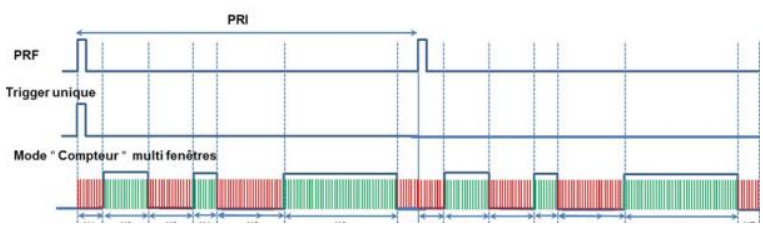

Fig. 4. Multi-windowing system implemented on the FPGA

\section{B. IF-Digitizer architecture and performances}

This digitizer architecture is quite simple as on Fig.5

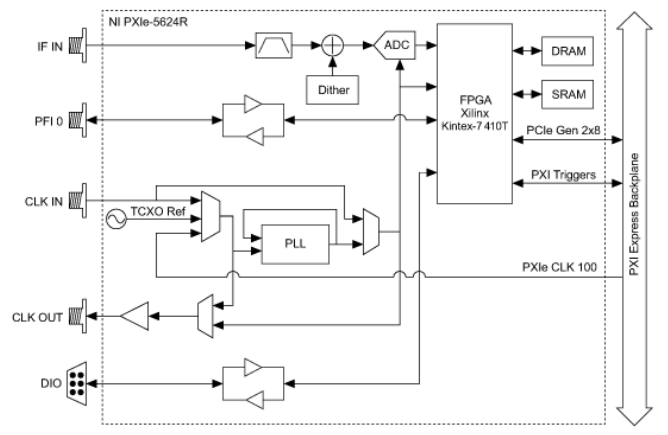

Fig. 5. NI PXIe-5624R IF-Digitizer

We are almost directly connected from the IF IN to the single ADC with real sampling at $2 \mathrm{GS} / \mathrm{s}$. All the processing is done in real-time on the FPGA, like calibration, phase synchronization between cards, digital down conversion, trigger handling, etc. There is no conditioning system inside, analog Local Oscillator (LO) or amplifier. so that your system needs to be adapted to fit within the frequency and amplitude of this digitizer.

Fig. 6 shows some Spurious Free Dynamic Range (SFDR) measurements provided by National Instruments. This specification is one of the most important in SAR imaging because it directly impacts the image quality.

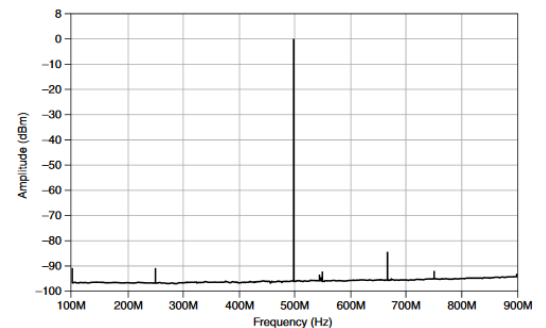

Fig. 6. Single-tone spectrum, $800 \mathrm{MHz}$ instantaneous bandwidth

The full bandwidth for this digitizer offers a SFDR ranging from $-80 \mathrm{~dB}$ to $-70 \mathrm{~dB}$.

\section{Vector Signal TRANSCEIVER (VST)}

PXIe-5840 is a Vector Signal Transceiver; it can be used as a RF waveform generator and RF signal analyzer, at the same time. It is the second version from National Instruments.

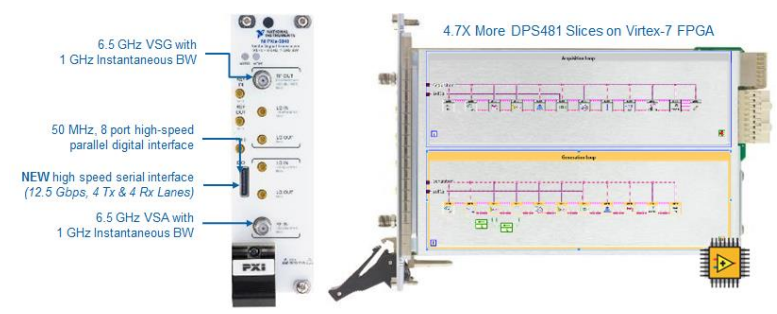

Fig. 7. Illustration of a NI VST and LabVIEW FPGA programming

It has $1 \mathrm{GHz}$ of real-time bandwidth both in generation and reception, between $9 \mathrm{kHz}$ to $6 \mathrm{GHz}$. An illustration of this VST is depicted on Fig. 7 and its simplified architecture is shown on Fig.8.

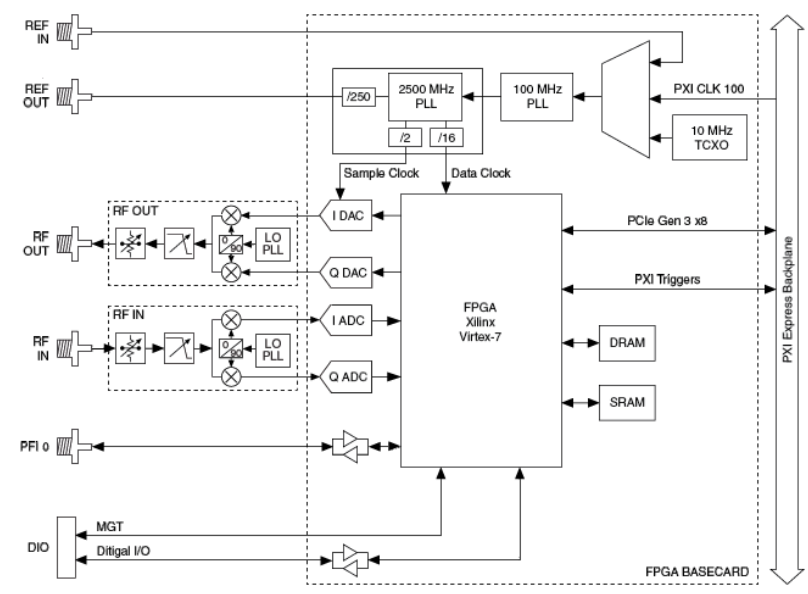

Fig. 8. VST architecture (simplified) 


\section{A. VST architecture}

As opposed to the IF-Digitizer, the VST is a complete RF instrument. The RF input and RF output have independent local oscillators, frequency coverage from $6 \mathrm{kHz}$ to $6 \mathrm{GHz}$ with homodyne conversion (Zero-IF). However, unlike a heterodyne generator or receiver, the frequency of the LO is identical to, or very close to, the frequency of the RF signal, resulting in a DC-centered signal.

The input signal is mixed down to baseband and split into in-phase (I) and quadrature-phase (Q) components. The I and $\mathrm{Q}$ path signals are then separately digitized resulting in I and $\mathrm{Q}$ data, which is equivalent to $2 \mathrm{ADCs}$ for only one RF signal. Finally, I and $\mathrm{Q}$ data streams are combined in software, rendering the original signal.

\section{B. Homodyne (Zero-IF) architecture advantages}

The homodyne architecture boasts a number of advantages over the traditional heterodyne architecture including simpler design, lower cost, less power consumption, and high selectivity, which allows separation of adjacent channels whose signals overlap. Other advantages include higher potential bandwidths, simpler designs with single LOs, and a smaller footprint due to a more compact design. These advantages are described in more detail hereafter.

- Bandwidth: Receivers with single ADCs have a practical upper limit for signal bandwidth of 40 percent of the sample clock frequency. With the same sample clock frequency, homodyne architectures allow doubling the bandwidth, or 80 percent of the sample clock frequency, because two ADCs are used. In general, ADCs with lower allowable sample clock frequencies have better SFDR and Signal-to-Noise Ratio (SNR) performance. Homodyne receivers allow wider bandwidths without the trade-off in ADC performance that is a necessary trade-off of single $\mathrm{ADC}$ receivers.

- Single LO: With multichannel measurement systems becoming more important for Multiple Input, Multiple Output (MIMO) applications, sharing the LO is a requirement. With only one LO to share in a homodyne architecture as opposed to multiple LOs in a traditional heterodyne architecture, homodyne architectures become more cost-effective and less complicated system to configure.

- Compact design: Homodyne architectures have much simpler RF designs over heterodyne architectures. Fewer LO signals; less expensive RF and IF filters; and fewer conversion stages for the homodyne architecture make for a more compact design.

\section{Homodyne (Zero-IF) challenges}

Although the advantages are numerous, the homodyne architecture does come with its own set of challenges, such as the DC offset reduction and signal image rejection. Common sources of error are shown on Fig.9 [2].

- LO leakage or offset: Any signal that mixes down to 0 $\mathrm{Hz}$ in the ZIF structure causes a spectral component at DC. This distortion falls at the center of the instantaneous bandwidth of the data acquisition and is retrieved as a DC offset term in the center of each data acquisition. Reducing the DC offset is accomplished on the digitized I and Q data streams by applying gain offsets.

- Signal image: With this architecture, a small difference between I and Q path can drastically degrade performances. In particular, phase skew and gain imbalance. The main solution to correct this is to use good quality components and the ability to calibrate the device and compensate skew and imbalance with digital filtering, for example. This is the way the VST works.

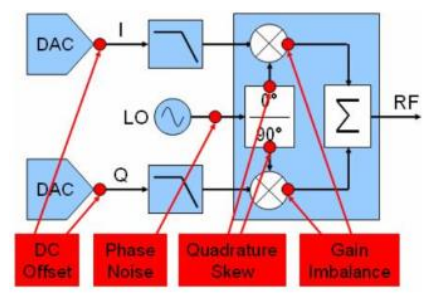

Fig. 9. Common sources of error in a Zero-IF Transmitter

As an example, the temporal cutoff of a large-band chirp is pictured on Fig. 10. The signal is generated through the VST and digitized with the IF-Digitizer. Two main effects appear on this picture due to VST and his ZIF architecture: a peak around $0 \mathrm{~Hz} @-77 \mathrm{dBm}$ and the signal image, due to phase skew and gain imbalance, between about $-400 \mathrm{MHz}$ and $-150 \mathrm{MHz}$.

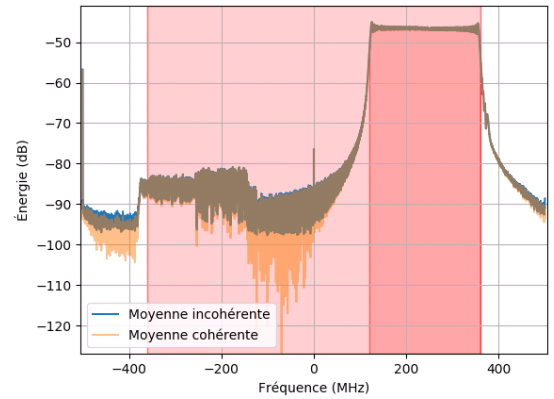

Fig. 10. Temporal cut-off of a chirp (120MHz to $360 \mathrm{MHz}), 800 \mathrm{MHz}$ instantaneous BW

\section{CONCLUSION}

Nowadays SAR systems are more and more complex. The Département ÉlectroMagnétisme et Radar (DEMR) of the ONERA aims to offer a large choice of measurements type, like different frequencies, multiple antennas, real-time signal processing... for various remote sensing applications. In order to adapt the system to a constant evolving technology and different operational configurations, the need of a reliable, flexible and always more powerful system makes the National Instruments platform and tools meet these goals.

\section{ACKNOWLEDGMENT (Heading 5)}

I would like to thank the entire DEMR-EXA and DEMRTERE teams.

\section{REFERENCES}

[1] G. Bonin and P. Dreuillet, "The Airborne SAR-System: SETHI," in Proc. EUSAR 2008, held in Friedrichshafen, Germany, 2008.

[2] National Instruments website, "Sources of Error in ZIF architecture" http://www.ni.com/tutorial/5657/en/ 